\title{
Risk reduction in dimension inspection of the plastic injection-molded parts from mechatronic devices by using optical 3D measuring techniques
}

\author{
Ion Cristian Braga ${ }^{1, *}$, Anisor Nedelcu ${ }^{1}$, and Razvan Udroiu $^{1}$ \\ ${ }^{1}$ Transilvania University of Braşov, Department of Manufacturing Engineering, Mihai Viteazu No.5, \\ Braşov, Romania
}

\begin{abstract}
As of the definition presented by Harashima, Tomizuka, and Fukada in 1996, the mechatronics is the synergistic combination of precision mechanical engineering, electronic control and systems thinking in the design of products and manufacturing processes. The most of the mechatronic devices need the precise dimensions of the plastic parts, as long as the combination of those parts leads to a final haptic characteristic defined within specific limits or when the certain travel way is linked with an electrical contact. The increasing of the risks to produce bad mechatronic devices are directly related to the combination of the plastic injectionmolded parts out of different cavities. The paper's aim is to present reducing of the risks to have bad final parts assembled with the components out of plastic injection-molded parts by using optical 3D measuring techniques at first validation of the parts out of the tool and setting parameters in the injection machines. The shrinkage and the warpage are more easily detected in that way and this will support first article inspection, but also during the entire production process. A case study presents the analysis of the data coming from the measurements of the plastic parts from each cavity and the combination of those parts, by using the ATOS inspection software. The CAD data are compared with the measured ones and the differences will be visible in the colored plotted areas, also the differences of the parts out of distinct cavities will be also displayed by overlaying of the measurements.
\end{abstract}

\section{Introduction}

The plastic material is more or less a modern material, even the first manufacturing of one kind of plastic, cellulose nitrate, known as Celluloid, was discovered in 1856 and refined in 1877. In the ninetieth century, Bakelite was the other plastic invented and used more in the manufacturing of the telephones or radios, the inventor being Leo Baekeland. The twentieth century, starting with 1935, the other inventions brought new materials as Styrofoam, polyvinyl chloride (PVC), acrylic, polyurethane, epoxy, and nylon, even new materials as synthetic rubber or textile fibers [1].

\footnotetext{
* Corresponding author: jbc69mc@gmail.com
} 
The automotive industry starts to use the plastic material in the $1950^{\text {th }}$ when the ABS was first used then polyamide and polycarbonate, also, alloys and blends of various polymers. Nowadays, a lot of automotive components are made from plastic materials, and can be mentioned the bumpers, interior trim, seating, dashboard, exterior trim, under-bonnet components, lighting, liquid reservoirs, fuel systems [2].

The challenges are bigger now when the future of the automobile is focused on reducing the $\mathrm{CO}_{2}$ emissions to reach limit $95 \mathrm{~g} \mathrm{CO}_{2} / \mathrm{km}$ in 2020, so the reducing of the weight become a priority. And this reduction of the weight can be obtained by replacing the actual materials, like metal, with plastic materials, because almost $75 \%$ of all materials used in manufacturing of the automobile are metal, as a study published in the magazine of the Plastics Europe AISBL, based on the study of Association Française de Mécanique (figure 1).

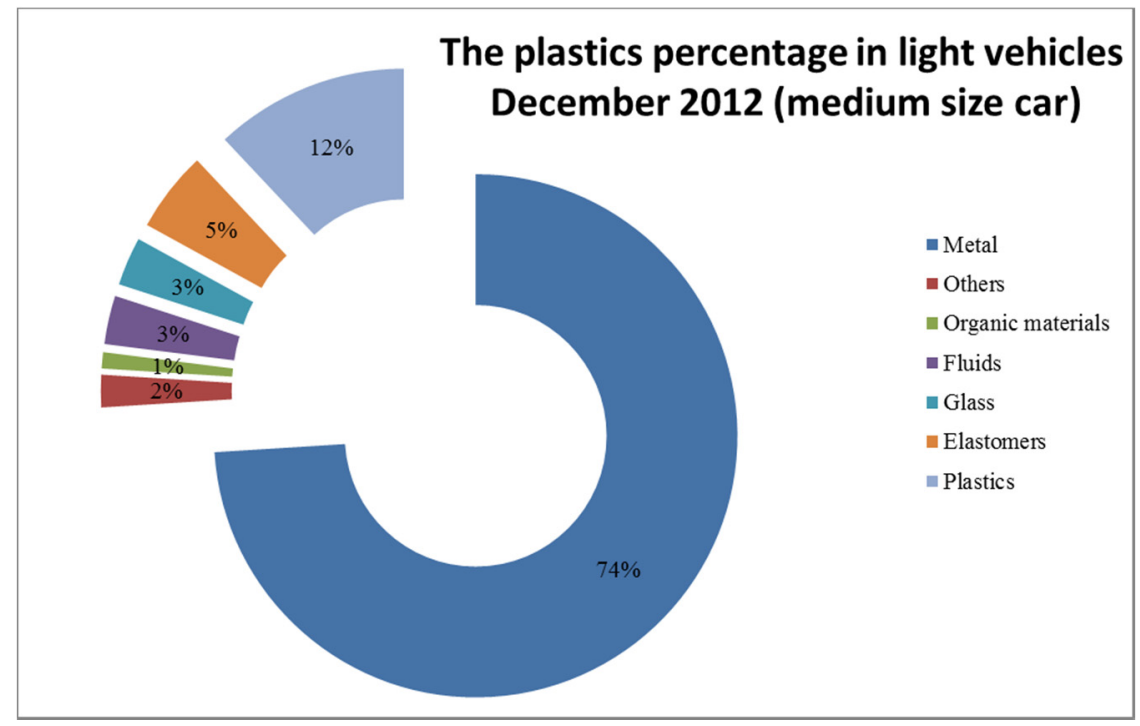

Fig. 1. Material percentage in light vehicles.

The same magazine, using the data from 2012, presents also the percentage of the using for the plastic materials in the automobile, under four categories: interior, exterior, under the hood and electronics (figure 2). As in interior a lot of mechatronic devices are used in order to realize the comfort of the driver and the passengers, as well as the safety aids using the sensors, buttons and touch screens [3], the parts manufactured by plastic injection molded processes need the precise dimension to fit with the other components and to reach the specific goals of each one.

And this precision dimensioning of the plastic parts will be obtained by early development of the product and the processes, but also in the serial production. The serial production processes of the plastic injection molded parts must be checked at the start of production and the validation of the injection molding machine and the molding tools must be validated prior to this. Of course, also the working methods and the training of the people who adjust or operate the machine are to be made prior. Then the monitoring of some dedicated characteristics will ensure that the process is stable and the outputs are according to the expectation.

This paper presents the possibility to reduce the risks in the serial production of the plastic injection molded parts by using the optical 3D measuring techniques at validation of the start of the production of a batch, and not using only the usual 2D measurement equipment as caliper or dial indicator or microscope. 


\section{Validation of the plastic injection molding processes}

\subsection{The plastic injection molding process}

The plastic injection molding processes is a repetitive process using the plastic material, usual pellets or granules, which is melted and heated with the reciprocating screw into a barrel and injected into a mold cavity through a nozzle, then held under pressure until is removed in a solid state and ejected.

The mold tool could be with one or more cavities, which can be similar cavities or not. This mold is made from two parts: one is fixed where the material is injected and the other is moveable, on the moveable platen, as is presented in figure 2 [4].

Generally, there are two types of the plastic materials molded: the thermoplastics and the thermosets. The thermoplastics, more often used, can be melted and solidified in a repeating cycles, heated at around 260 Celsius degrees. On the other hand, the thermosets are heated on a temperature around 120 Celsius degrees, upon their final heating, become insoluble and infusible permanently [5]

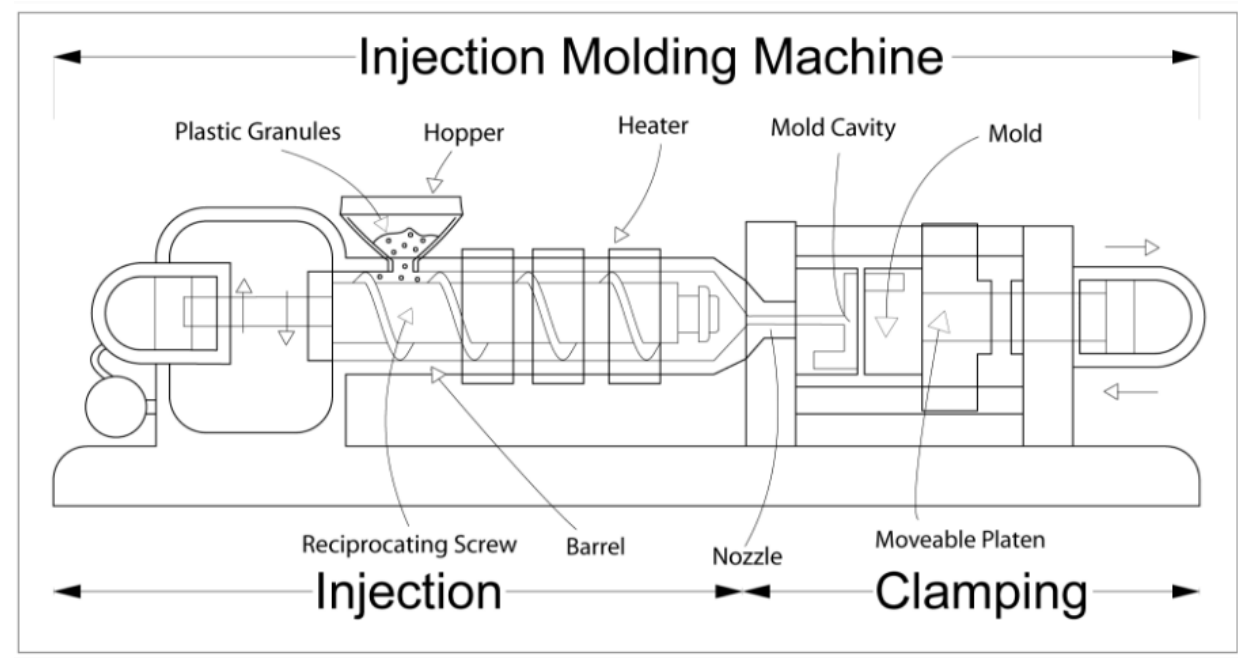

Fig. 2. An injection molding machine.

Many factors can influence the product dimension tolerances and are very important to keep these factors under control. Can be mentioned here the design of the product, the plastic material, the design of the mold, the capability of the injection molding machine and the process parameters such as cooling temperature, demolding temperature, mold temperature, melt temperature, injection time, holding time, mold close or open time, injection pressure, back pressure or holding pressure.

\subsection{The validation of the plastic injection molding process}

Even the design of the product and the mold will take care to prevent the shrinkage or the warpage on the parts, by choosing the location of the gate and the material selection [6], the issues can be occurred also in the serial production and these effects will generate issues in the next assembly processes of a mechatronic device. Therefore, the selection of the process parameters depend on the type of the injection machine used, and the setting operator will 
work with some changes of these parameters, at the end the product can have deviation from the expected dimensions.

In order to prevent this, after the validating of the product design and the validation of the machine capability, it is necessary to validate the injection molding process at each start of production and to monitor some selected characteristics along the entire batch production. The statistical process control can support the team to detect the variation in the process and the trend of the processes, in that way to prevent to produce parts put of tolerance and to generate the scrap or bad assembled mechatronic devices.

\section{Study case}

\subsection{The validation of the start of production by using 1D/2D measurement devices}

For the study case was selected a part from a mechatronic device manufactured in the processes of the plastic injection molding, a cover used afterwards in an assembly where will have some movements in the functioning, a rotation movement in a combination with a housing (figure 3 ).

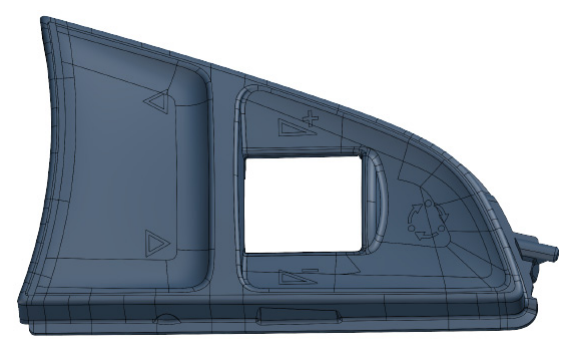

Fig. 3. A plastic injection molded cover.

The dimension inspection of the part at the start of production and in the monitoring visual failures at each four hours is made by measurement of a characteristic, dimension K1, with a dial indicator in the laboratory, by the operator, as in figure 4, but also by visual checking of the part to detect the issues as incomplete molding, sink mark, scratches, burrs or other visual failures.

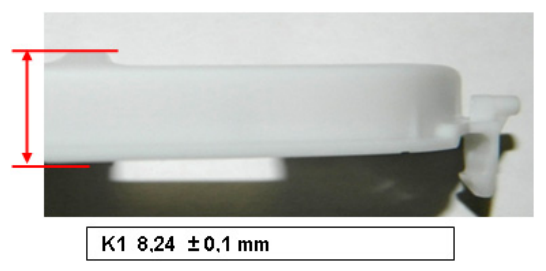

Fig. 4. Measuring method for the characteristic.

The dimension inspection of the part at the start of production and in the process monitoring at each four hours is made by measurement of a characteristic, dimension $\mathrm{K} 1$, with a dial indicator, in the laboratory, by the operator, as is presented in figure 4, but also is made by visual checking of the part to detect the possible issues as incomplete molding, sink mark, scratches, burrs or other visual failures. 
Based on the measurements of the $\mathrm{K} 1$ with the dial indicator and referring to the specification, the sample parts are within the tolerances, the same the attributive visual characteristics. In order to monitor the variability and stability of the process, the capability of the machine was evaluated using the software Qs-Stat, Machine Acceptance $\mathrm{Cm} / \mathrm{Cmk}$, from Q-Das [7], and the result shows a very good machine capability index, Cmk, and the model distribution being a normal distribution (figure 5).

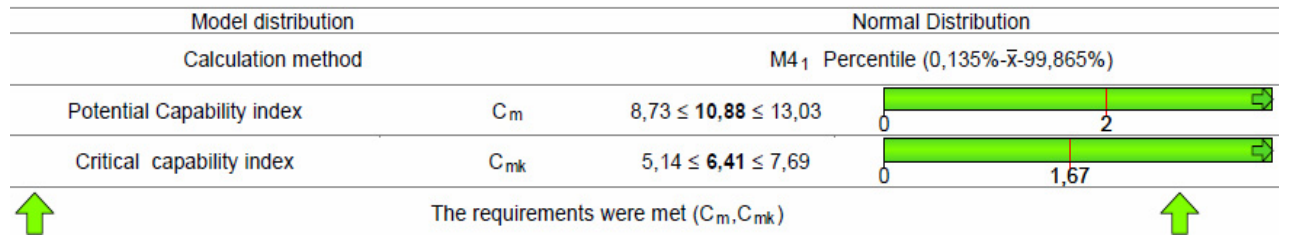

Fig. 5. Capability index and model distribution.

The Gauss curve shows a narrower distribution, as in figure 6, it mean, the process is precise enough. At the end, the decision made on the sampling is that the serial production is according with specification and parts can be used in the next steps in the processes, and there are not detected any risks in link with the functioning of the entire mechatronic device.

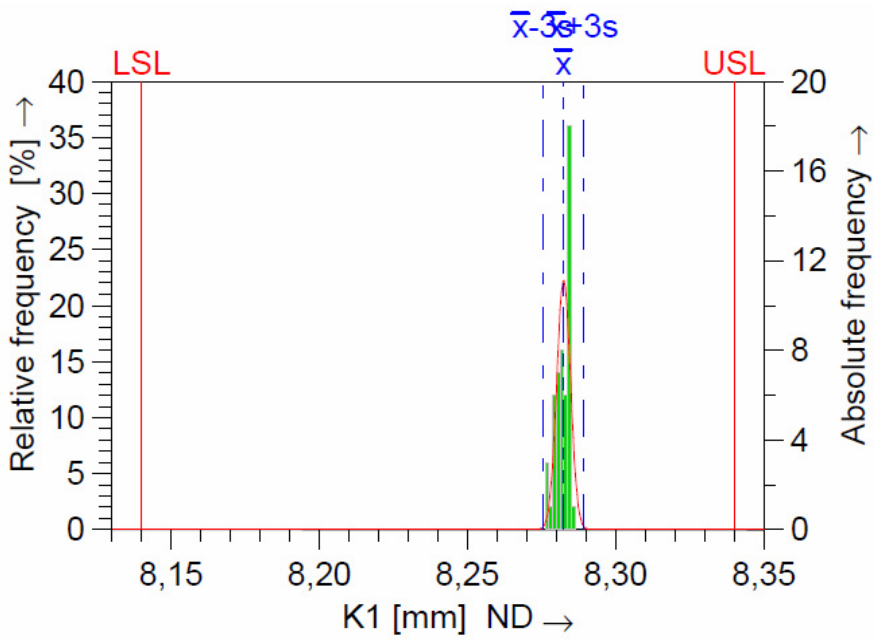

Fig. 6. Gauss curve.

As was mentioned in the chapter 2.2, from the design of the product and design of the mold was considered the warpage limitation, but also the selected process parameters can influent the final dimension of the part and the warpage or even shrinkage could occur, and there could be some supplementary studies by using Taguchi method to establish the optimal combination of the process parameters [8]. And the 1D/2D measurement devices and the measurement system established only on these measurements, even combined with the statistical process control will not secure enough all the possible risks to deliver the plastic injected molded products in the assembly and there to detect some issues from functioning of the mechatronic device. 


\subsection{The validation of the start of production by using optical 3D measuring technics}

In the above paragraph was presented the possible risks, not detectable with the measuring system established, and the probability of generating scrap in the assembly processes can be considerable. Because of that, the study case presents the using of the optical 3D measuring technics.

The paper proposes to design a combined measuring system based on the $1 \mathrm{D} / 2 \mathrm{D}$ device measurements to monitor the process through entire batch using statistical process control, but validating the start of production of the batch by using optical 3D measurements.

Hence, the possible shrinkage or warpage could be easily detected by the appraisal and the quick decision will lead to checking of the parameters and optimizing them so that the parts will be free of these kinds of issues.

\subsubsection{Optical 3D Coordinate Measuring Machine}

The 3D Coordinate Measuring Machine is an optical 3D measuring machine developed for a quality control in the production and manufacturing process. More models are available for different part sizes and applications, the case study used ATOS ScanBox - Optical 3D Coordinate Measuring Machine, Series 4, ATOS CORE MV 135.

The core of the systems is the 3D scanner, whose sensors provide full-field 3D coordinates for each individual measurement and up to 16 million independent measuring points are captured within some seconds. The scanning system is based on the triangulation principle: the sensors projects different fringe patterns over the parts to be measured, then two cameras will record this, as in figure 7.

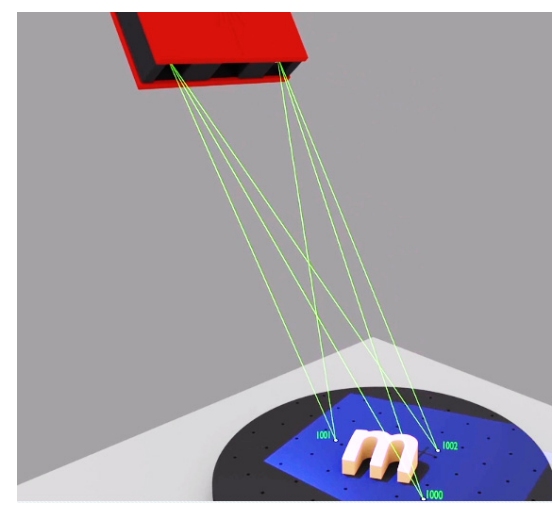

Fig. 7. Measuring principle: fringe and triangulation.

The visible points seen by both cameras in a single scan are recorded and in order to have complete measured part digitized, the different angle measurements will be done. Some reference circular points which are fixed on the frame for part fixation, as in figure 8 , are used in order to transform each measurement into the global coordinate system [9]. 


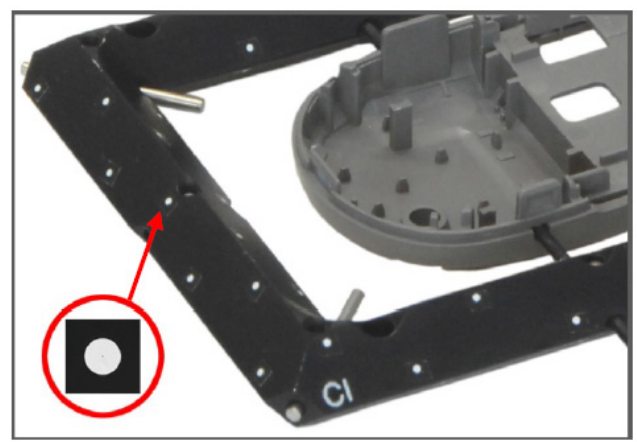

Fig. 8. Measuring principle: reference circular points.

\subsubsection{Comparing the scanned part with the CAD model}

The case study is made on a part coming out from a mold with 2 cavities. The first measurement will have the aim to compare the scanned result of the part with the 3D model from CAD. In figure 9 is presented the overlapping of the measurement from the scanned part with this CAD model. As can be seen, in the upper right corner and in the lower left corner are some deviation from the model and there the warpage is present on the measured part: in the left lower corner, are two points in blue colour, 0.079 and 0.063 , the sign minus in front of the points shows that the part seems to have less material than the requirements. In the same way, it can be seen in the right upper corner another two points in blue colour, 0.071 and 0.048 . Based on the assembly tests and the studies for the combination of the cover with the other assembled parts (housing, electronic, rubber etc) the assembly processes could be approved or not, depend on the results positive or negative of the tests.

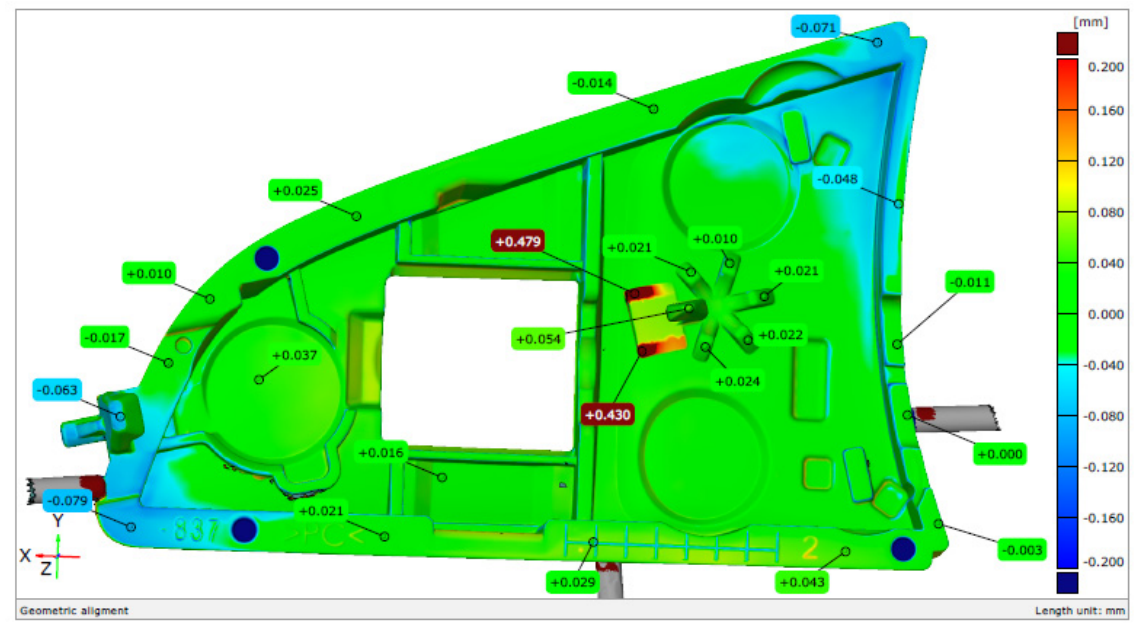

Fig. 9. Scanned part cavity 2 versus 3D model.

\subsubsection{Comparing the scanned part from cavity 1 with the scanned part from cavity 2}

While the measurement by comparing the scanning with the CAD model shows to the assessor the areas where the part is out of tolerance or even not out of the tolerance but warpage or shrinkage detected, by comparing the scanning of the part from cavity 1 with 
scanning of the part from cavity 2 will let the assessor to take more quick the decision if the assembly test for the validation of the start of the production must include all the combination between the cavities for all assembly components (figure 10). As can be seen in this picture, there are some very small deviation between the parts from cavity 1 versus cavity 2 , therefore, the parts are almost identical and the decision is to be ordered only a sample using one cavity for the following assembly tests. It will save money and the risks to produce scrap in the next step are prevented, also, the cost of quality prevention will be decreased, thanks to a decision made on the comparison between the cavities.

And the reducing of the prevention cost will be as bigger as numbers of injected molded parts are coming from a mold with more cavities. For instance, based on the samples from the presented study, there can be seen that are almost any differences between both cavities, so for the assembly tests is enough to use one of the cavities and, if the detected warpage on the corners will not lead to a malfunction, the plastic injection molding process could be approved and the serial production of the batch could start and run until some possible specific deviation detected in the statistical process control monitoring.

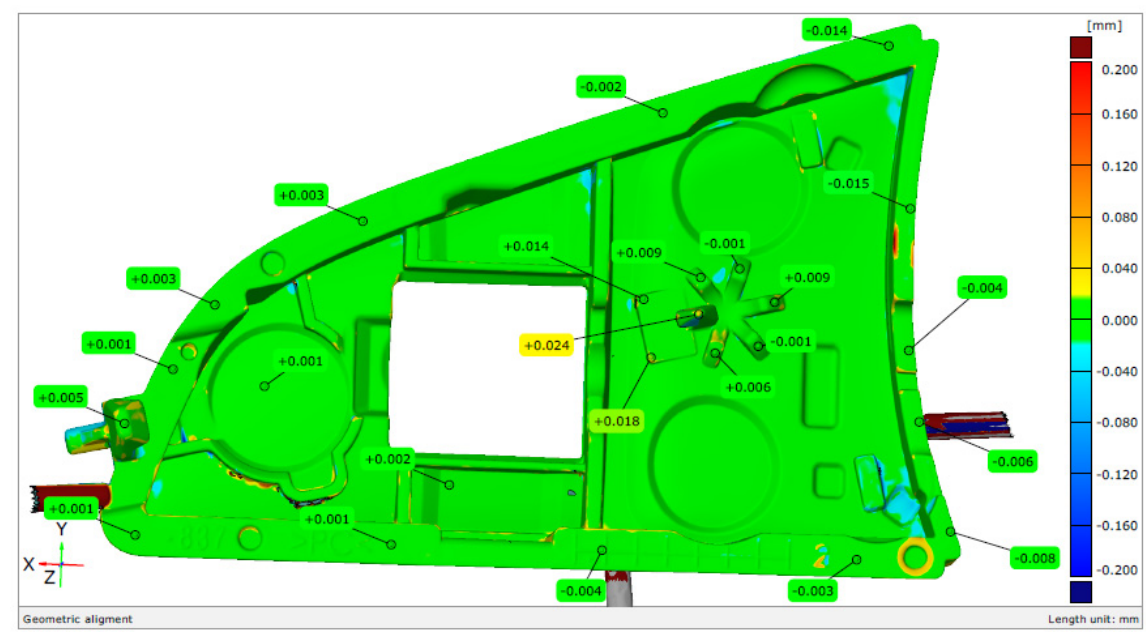

Fig. 10. Scanned part cavity 2 versus part cavity 1 .

\section{Conclusions}

Nowadays, in almost all of the organizations which have the plastic molding injection processes, the method used to validate the start of production is by choosing some characteristics, then measuring these characteristics with 1D/2D measurement devices and follow up the capability of the process. Only at validation or revalidation after repairing and maintenance of the mold it is used the 3D optical measurements

The proposed method to use the optical 3D measuring in the dimension inspection of the plastic injected molded parts at starting of the production of each batch will prevent the risks to produce parts affected by warpage or shrinkage, even the selected characteristics to be measured shows good result and the statistical process controls shows very precise, based on the distribution and capability index.

Another advantage of this method is represented by the saving of the quality prevention cost through comparing the scanned parts coming from different cavities of the same mold or even different molds, also the saving of the rework cost from next step operation in assembly processes. As the method is more precise and accurate, could be used also in the 
other inspection areas and to replace the conventional measuring system or to be used complementary at validation of the processes.

\section{References}

1. R. Mustalish, Modern Materials: Plastics. In Heilbrunn Timeline of Art History. New York: The Metropolitan Museum of Art (2000)

http://www.metmuseum.org/toah/hd/mome/hd_mome.htm (2004)

2. K. Szeteiova, Automotive Materials: Plastics in Automotive Markets Today, Slovak University of Technology, (Bratislava, 2010)

3. Automotive - The world moves with plastics http://www.plasticseurope.org/Document/automotive---the-world-moves-with-plasticsbrochure.aspx?Page=DOCUMENT\&FolID=2 (2013)

4. AV Plastics, http://www.avplastics.co.uk/what-is-injection-moulding (2016)

5. D.V. Rosato, M. G. Rosato, Injection Molding Handbook, (Springer Science+Business Media New York, 2000)

6. M. Huszar, F. Belblidia, H. M. Davies, C. Arnold, D. Bould, J. Sienz, Sustainable injection moulding: The impact of materials selection and gate location on part warpage and injection pressure, Sustainable Materials and Technologies 5 (2015)

7. Q-Das, Experst in statistics, http://www.q-das.com/en/applications/ (2016)

8. P. Postawa, J. Koszkul, Influence of processing conditions on changing of shrinkage and mass POM injection molding parts, COMMENT, (Gliwice Poland, 2005)

9. GOM, http://www.gom.com/metrology-systems/atos-scanbox.html (2016)

10. R. H. Bishop, The Mechatronics Handbook, (CRC Press, 2002) 